\title{
One year of Nature Catalysis
}

As we celebrate our first birthday, we thank everyone involved and look back on an exciting 12 months.

W ith this issue, Nature Catalysis celebrates its first birthday, and what an exciting, busy and productive 12 months they were! It has been a pleasure to read and publish such an array of top-quality science from across the entire field of catalysis. And we would like to open by saying a huge thank you to all our authors, reviewers and readers who helped make this year such a success.

In 2018, we published a total of 102 primary research articles. These cover areas from pure theory to practical advances, and from reducing oxygen to resurrecting ancient proteins. Anything in the broad field of catalysis that makes a conceptual leap or that shows a useful practical improvement is considered. For those interested in statistics, the breakdown in article numbers per subject area was as follows; electrocatalysis, 32; heterogeneous, 27; homogeneous, 21; photocatalysis, 7; and biocatalysis, 15. Interestingly, the relative size of each area published mapped very closely to the breakdown of the subjects of all submitted papers, meaning that no one area was especially favoured or disfavoured by editors or reviewers. Many of these articles were highlighted and explained to a broader chemistry audience by means of News \& Views articles - 39 in total, for those of you still counting - all written by independent experts in the field.

In addition to the primary research, we have a range of Reviews, Perspectives and Comments that explain and summarize important fields, or that gave authors a chance to give their opinion on something close to their heart.

Outside of papers we have also been busy ensuring that the content reaches as wide an audience as possible. Getting indexed in major databases (including Web of Science, Scopus and Chemical Abstracts) at a very early stage in our development was very heartening and a big boost in terms of discoverability. Social media has also been important, and we have ensured that every

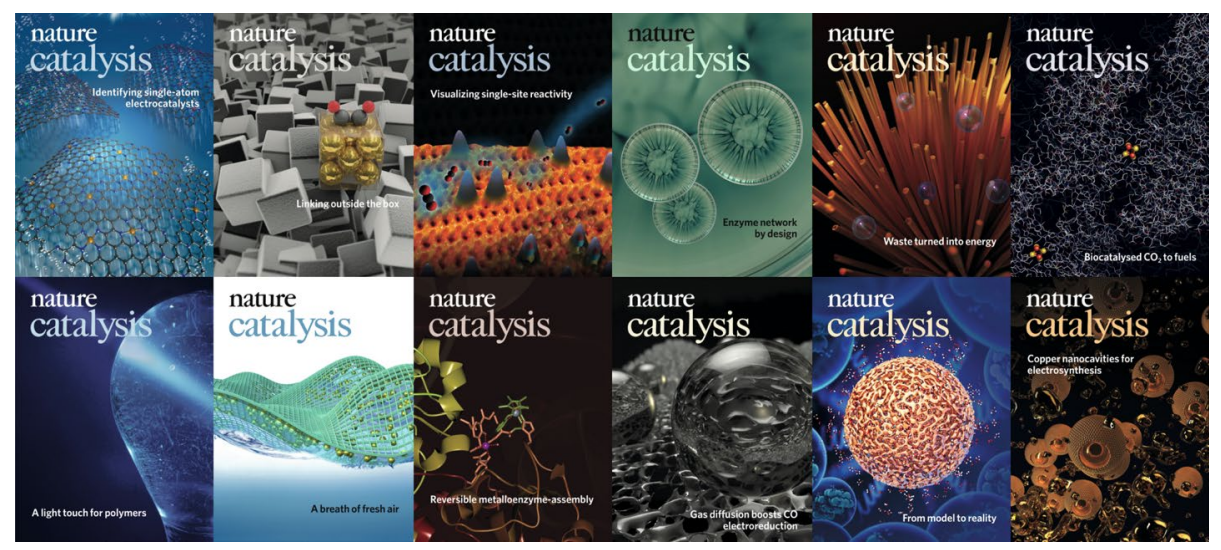

paper we publish is highlighted on our Twitter account (check out @NatureCatalysis for more). The fact that these posts are normally seen by about 10,000 people shows how useful this can be in getting your research noticed. And if you have not done so already I recommend checking out the chemistry blog from the Nature Research community (https://chemistrycommunity. nature.com/). The 'Behind the Paper' posts deserve a special mention as they provide an author's-eye-view on the background and history behind each finished article.

And now we look forward to another exciting year in 2019. Our January issue starts things off as we mean to continue with a selection of great science, both in the research and review sections. Check out the research on virtual screening of enantioselective catalysts, allowing users to identify ligands that are likely to perform well before they even start in the lab. A separate Comment looks at this same problem from a different angle: namely, how to go about experimentally screening catalysts with high-throughput methods. Sticking with synthetic chemistry, we have a study piecing together the route from $\mathrm{CO}_{2}$ to heterocycles, an article on the use of electrochemistry in asymmetric catalysis, one about an inexpensive and reusable nickel catalyst for reductive aminations and a Perspective on amide formation reactions from a green chemistry viewpoint. Biosynthesis also features with a Review - and a map - on the bio-production of industrially useful chemicals. Direct observation of alloy nanoparticles during $\mathrm{CO}$ oxidation shows the dynamic nature of the active species. And we wrap up with a trio of papers on quite different aspects of $\mathrm{CO}_{2}$ reduction; linking active sites to products in electrocatalytic reduction, a theory-driven catalyst development for electroreduction at low overpotentials and a demonstration that silicon-hydride nanosheets can be turned into catalytic reductants by the addition of palladium.

So, with that we thank you once again for your support in 2018, and look forward to exciting times in the field for the coming year.

\section{It has been a pleasure to read and publish such an array of top-quality science from across the entire field of catalysis.}

Published online: 14 January 2019 https://doi.org/10.1038/s41929-019-0228-4 